\title{
Subpattern formation during condensation processes on structured substrates
}

\author{
C. Schäfle, P. Leiderer and C. Bechinger \\ Fachbereich Physik, Universität Konstanz - Fach M676, D-78457 Konstanz, Germany
}

(received 24 February 2003; accepted in final form 26 May 2003)

PACS. 68.03.Fg - Evaporation and condensation.

PACS. 68.08.Bc - Wetting.

\begin{abstract}
We investigate the temporal and spatial development of condensation patterns on chemically patterned substrates. We find that droplets condense preferentially on lyophilic sites and are surrounded by a depletion zone where no further nucleation occurs. The size of the depletion zones can be tuned by the flow rate of the incoming gas stream. If the size of the depletion zones is in a suitable range, well-defined droplets grow on the interstitial sites of the lyophilic lattice, i.e. on the non-structured areas. This can lead to ordered substructures.
\end{abstract}

Introduction. - When supersaturated vapors are exposed to cold substrates, condensation starts and characteristic adsorbate patterns develop. This situation is encountered, e.g., during deposition of metals and semiconductors in PVD (physical vapor deposition) processes, but also in the case of condensation of water vapor on solid substrates. In the latter case the resulting condensation patterns are also known as breath figures [1]. In general, this phenomenon is explained as a heterogeneous nucleation process under constant flux conditions of molecules towards the surface and has been studied in detail during recent years [2].

In case of breath figures on homogeneous lyophobic substrates four different regimes can be distinguished and the following scenario is proposed [1-3]: after nucleation and growth of small embryo droplets, neighboring droplets start to coalesce when their contact lines touch each other. In this regime, the developing droplet patterns are self-similar with respect to, e.g., the droplet radii. In the third regime, a new generation of droplets nucleates and grows between the coalesced droplets of the first generation. Finally, the droplets are deformed by gravity when they exceed a typical size being comparable to the capillary length of the liquid, where gravitational effects become important.

These condensation patterns will be modified if the wettability of the substrate is increased [4]. In this case pinning of the contact line becomes important and the droplets tend to form nonspherical islands, although still a self-similar growth regime exists. Pinning even balances a slight wettability gradient so that the droplets remain motionless. The presence of a wettability gradient can only be observed during the coalescence of very large droplets, when the center of mass moves towards the lyophilic region. As the contact angle tends to zero, the liquid forms a quasi-film-like structure. 

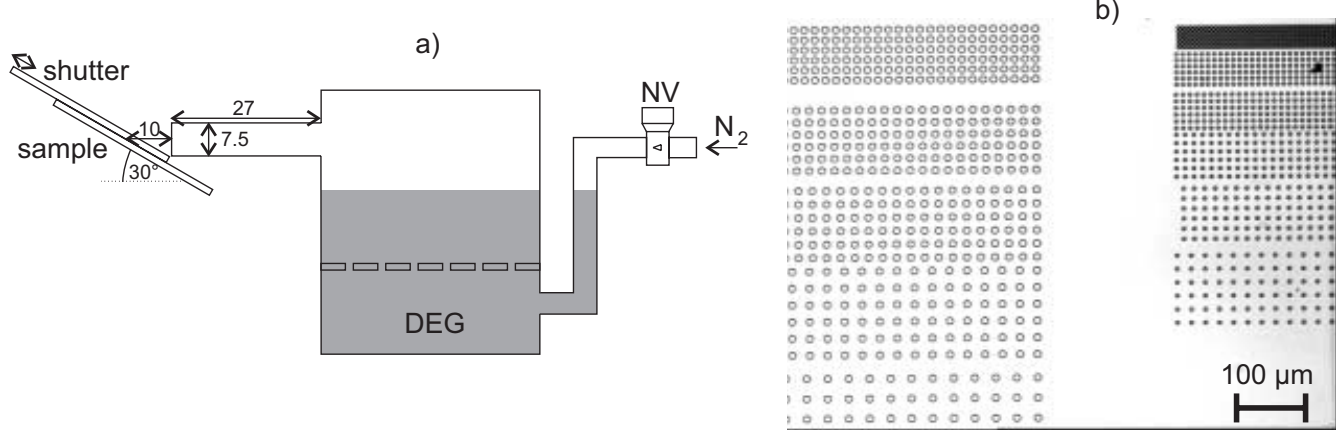

Fig. 1 - a) Experimental setup to produce a uniform vapor. b) Structure of the substrate: quadratically ordered circles with left: diameter $d=10 \mu \mathrm{m}$ and lattice constants $a=15,18,20,25,30$ and $40 \mu \mathrm{m}$; right: $d=5 \mu \mathrm{m}$ and $a=6,9,10,12.5,15$ and $20 \mu \mathrm{m}$.

Even more pronounced differences in the condensation process are observed on patterned substrates, which can be conveniently produced by microcontact printing $(\mu \mathrm{CP})$. It has been shown in this case that the droplets condense preferentially at the lyophilic sites $[5,6]$. Furthermore, Aizenberg et al. [7] demonstrated that when such patterned substrates are exposed to a supersaturated calcite solution, preferential nucleation at the patterned regions occurs. In addition, a depletion layer is formed around each nucleation site where essentially no nucleation and growth is observed. The origin of these depletion zones is explained in terms of a local reduction of the calcite concentration in the solution around the patterned regions. When this concentration is below a certain threshold, no further nucleation occurs.

In this work we investigate condensation patterns on chemically structured surfaces. Similar to the work of Aizenberg et al., we also observe depletion zones around lyophilic sites of the substrate. In addition, however, we also demonstrate that if the size of the depletion zones is in a range where they partially overlap, small droplets condense on the intermediate sites of the structures. This eventually leads to a regular subpattern where droplets are - contrary to what is typically achieved during $\mu \mathrm{CP}$ - formed at well-defined positions at unpatterned regions of the substrate.

Experimental preparation. - In order to create a uniform gas stream, which is highly saturated with liquid, we used a typical breath figure setup, as shown in fig. 1a; cf. [2,3]. A stream of nitrogen gas is controlled by a needle valve (NV) and first flows through a copper cell filled with a liquid and is then directed at an angle of $30^{\circ}$ onto the sample by means of a short tube with an inner diameter of $7.5 \mathrm{~mm}$. As liquid we have chosen diethlyene glycol (DEG), which was kept at a constant temperature of $68.0 \pm 0.3^{\circ} \mathrm{C}$ because it has a very low vapor pressure $\left(p=0.01\right.$ mbar at $T=25^{\circ} \mathrm{C}[8]$ ) and thus increases the lifetime of the condensation patterns. The gas flow was calibrated by the measured displacement of a certain volume per time.

Prior to a typical condensation experiment, the sample is first blocked with a shutter until a stationary gas flow is established. Then, the shutter is opened parallel to the substrate for a certain amount of time to allow gas to condense on the sample. After closing the shutter, the sample is immediately characterized by optical microscopy (bright field, reflection). Between closing the shutter and taking the video images around $30 \mathrm{~s}$ pass. During this time, the droplet pattern of the diethylene glycol changes only slightly.

Surfaces with well-defined quadratically ordered lyophilic circular patches are prepared by microcontactprinting $(\mu \mathrm{CP})$ of alkanethiols $[9,10]$. As substrates we used thermally evaporated 
gold films (2 nm Ti, $20 \mathrm{~nm} \mathrm{Au}$ ) on glass on which a $1 \mathrm{mM}$ ethanolic solution of $\mathrm{HS}\left(\mathrm{CH}_{2}\right)_{17}-\mathrm{CH}_{3}$ was stamped. This was achieved with a topographically structured stamp made from PDMS (Sylgard 184, Dow Corning), which was cast from a structured silicon wafer. This results in a well-defined lyophobic environment around the lyophilic patches. In some cases the circles were made more lyophilic by immersing the substrate in a $1 \mathrm{mM}$ ethanolic solution of $\mathrm{HS}\left(\mathrm{CH}_{2}\right)_{11}-\mathrm{OH}$ or $\mathrm{HS}\left(\mathrm{CH}_{2}\right)_{11}-\mathrm{COOH}$, rinsing it with ethanol or isopropanol and drying it under a nitrogen stream. Using $\mathrm{COOH}$-terminated thiols, one can obtain nearly completely wettable lyophilic areas if a subsequent rinse with a $6 \mathrm{~N} \mathrm{NaOH}$ solution and water is followed [11]. As a result, the contact angle of the lyophobic region is also lowered from $\theta \approx 69^{\circ}$ to $\theta \approx 45 \pm 5^{\circ}$. The lyophobicity can partly be regained by ultrasonification. With an $\mathrm{OH}$-termination the contact angle of the lyophobic area remains constant.

In order to compare the influence of the substrate geometry on the morphologies of the condensation patterns, we used a stamp consisting of several areas of quadratically arranged lyophilic circles with 5 and $10 \mu \mathrm{m}$ diameter and different lattice constants. We used different lattice constants to study isolated droplets on lyophilic sites and the increasing influence of neighboring ones. Figure $1 \mathrm{~b}$ shows an overview of the stamp. As can be seen, the different patterns are very close to each other, and it can be assumed that the gas flow is constant for all different periodicities. This means that the condensation patterns on different parts of the sample are directly comparable in one experiment. Attention was paid that the wetting structures are always stamped on the same place of the samples. Therefore, the structure was always exposed to the same region in the middle of the gas flow and led to reproducible patterns. For each adsorption experiment a freshly prepared sample was used.

Results and discussion. - In a typical condensation pattern experiment we observe that randomly distributed droplets develop in the homogeneous lyophobic regions of the substrates (contact angle $\theta \approx 69^{\circ}$ ). They grow and coalesce in a similar way as has been described by other authors [1]. On the contrary, a chemical structure on the substrate can induce a local ordering in the condensation patterns. In fig. 2, a typical example of such a droplet pattern around an isolated lyophilic circle can be seen. The liquid condensed preferentially on the lyophilic patch and formed a large droplet, which covers the whole lyophilic area. This large droplet is surrounded by a well-defined region, where no nucleation and growth of droplets occurs. This zone is the after-mentioned depletion zone and will be the subject of the following study. Outside this region, the density of small droplets is high. The remaining part of fig. 2 will be explained below.

The sizes of the depletion zones at constant experimental parameters (temperature of the liquid, gas flow, position of the sample in the gas flow) are well reproducible. Their size is measured by measuring the radial distribution function around a large droplet with an image processing program. On the same substrate the sizes of the depletion zones around each droplet are practically identical. Their nominal standard deviations are around $0.15 \mu \mathrm{m}$. However, between different samples in a series of experiments the variations are slightly larger. This is taken into account by the error bars of fig. 3, which show the measured depletion zones around the hydrophilic regions for different flow rates. The triangles and squares correspond to $5 \mu \mathrm{m}$ and $10 \mu \mathrm{m}$ lyophilic patches, respectively. One clearly sees that the size of the depletion zones decreases in both cases as a function of the incoming gas flow.

The size of the depletion zones is also found to depend on the wettability of the lyophobic region. Figure 4a shows an example of two samples, which are exposed to the same gas flux for equal times $(8.2 \mathrm{ml} / \mathrm{s}$ gas flux for $10 \mathrm{~s})$, but which have different contact angles (left $\theta \approx 69^{\circ}$ and right $\theta \approx 45 \pm 5^{\circ}$ ). The depletion zone is clearly smaller on the samples with the smaller contact angle. 

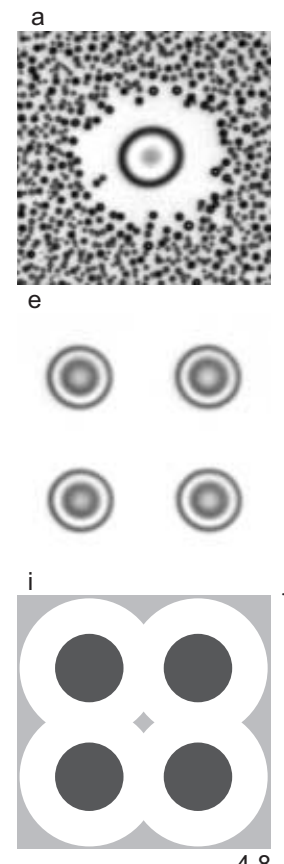

4.8
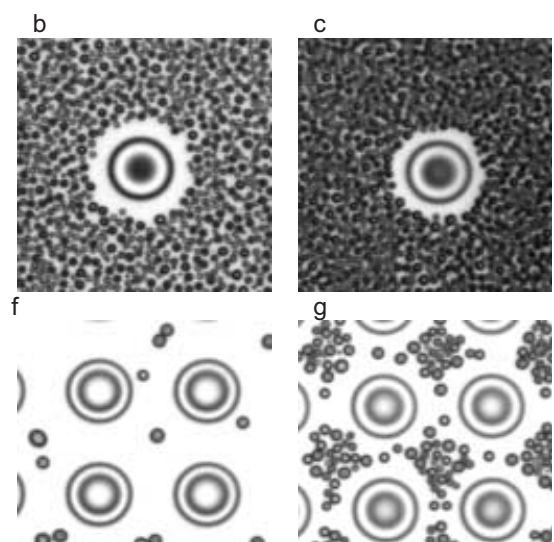

( j

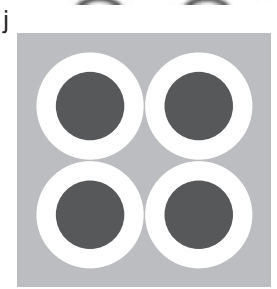

6.6

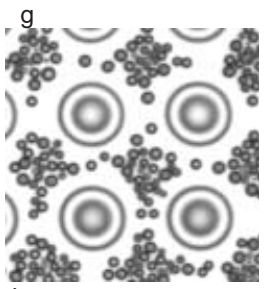
$\mathrm{k}$

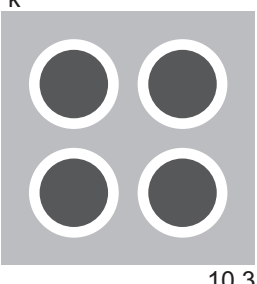

10.3
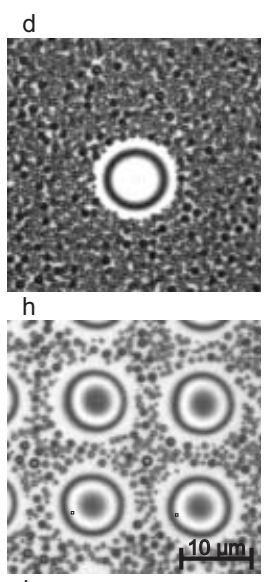

I

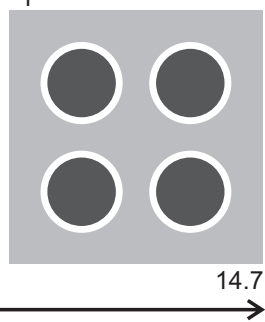

gasflow $[\mathrm{ml} / \mathrm{s}]$

Fig. 2 - Optical images of breath figures on substrates with circular patches of $10 \mu \mathrm{m}$ diameter on a lyophobic background. The circular patches are arranged in a square lattice. The flux of the incoming gas increases from left to right. a-d: single droplets on the lattice with the largest lattice constant $(40 \mu \mathrm{m})$. Around each droplet a depletion zone is observed which decreases with increasing flux. e-h: similar as before but with a lattice constant of $15 \mu \mathrm{m}$. At small flux the depletion zones overlap and no droplets can form in between the big droplets. With decreasing flux, again the depletion zones become smaller. i-l: schematic illustration of overlapping depletion zones on a square lattice with $15 \mu \mathrm{m}$ lattice constant. The extension of the depletion zones was taken from a-d. As can be seen in i, the depletion zones overlap almost completely and thus explain why no droplet nucleation in e occurs.

If the lyophilic patches are not completely wettable (e.g., if they consist of the bare gold surface), the liquid possibly covers the circle only partially. Nevertheless, the depletion zone has a round shape, which follows the chemical structure on the substrate (cf. fig. $4 \mathrm{~b}$, right image). This is in contrast to a droplet with a similar size, which is not localized on a lyophilic patch, but somewhere else on the substrate. Here, the depletion zone follows the contour line of the droplet. Therefore, we could presume that it is the lyophilic areas and not the droplets themselves that induce the formation of depletion zones.

Our observations are consistent with the following picture: the nucleation rate on lyophilic areas of a substrate is higher than on lyophobic regions. These regions act as sinks for diffusing molecules, where droplets condense and grow preferentially. This leads to a concentration gradient of diffusing molecules on the lyophobic areas on the substrate (cf. [12]). If the local concentration is below a critical value where nucleation occurs, the nucleation within a certain region around the droplet is inhibited. This leads to a depletion zone where no further nucleation occurs. The flux of incoming molecules determines the concentration gradient of diffusing molecules. Accordingly, the depletion zone becomes smaller with increasing flux. 


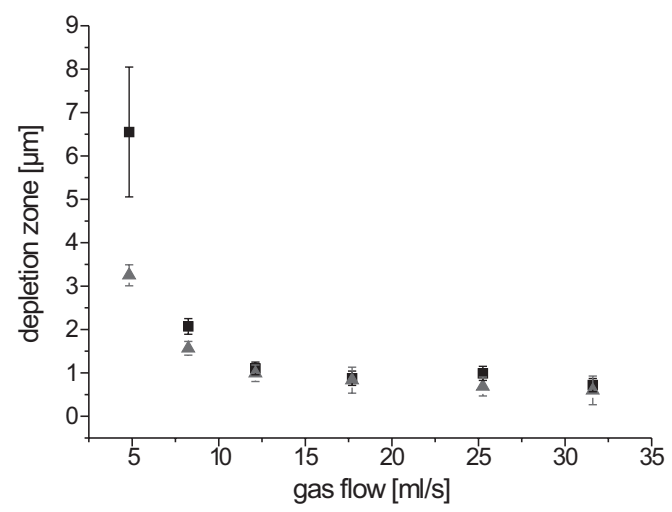

Fig. 3

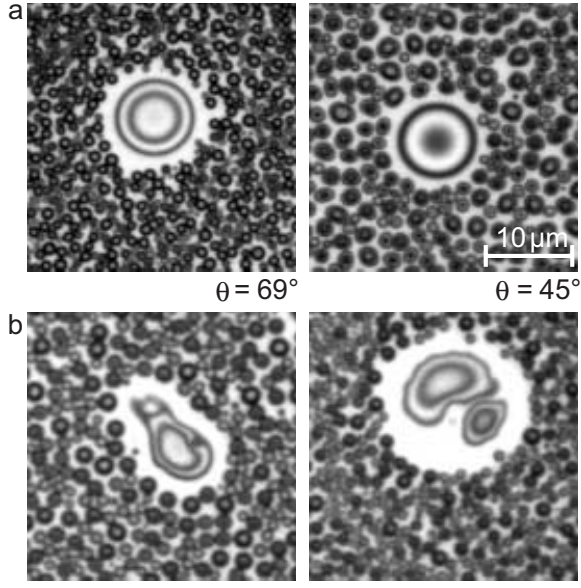

Fig. 4

Fig. 3 - Dependence of the width of the depletion zones on the gas flow around the lyophilic circles with $\Delta 5 \mu \mathrm{m}$ and $\square 10 \mu \mathrm{m}$ diameter. The contact angle of the lyophobic area is $\approx 70^{\circ}$.

Fig. 4 - a) Dependence of the size of the depletion zones on the contact angle $\theta$ of the lyophobic region; left: $\theta=69^{\circ}$, right: $\theta \approx 45 \pm 5^{\circ}$. b) Lyophilic circles acting as a sink for diffusing molecules; left: a big droplet in an unstructured region, the depletion zone follows here the contact line of the droplet; right: big droplet on lyophilic circle, the depletion zone follows the lyophilic circle.

On the other hand, the free-energy barrier for nucleation is lowered by a more lyophilic environment (see $[4,13]$ ) leading to smaller depletion zones.

So far, we only discussed the formation of depletion zones on isolated patches. Now we consider how the condensation patterns are influenced by neighboring large droplets and their possibly overlapping depletion zones. In fig. 2 the influence of the patterned substrate on the droplet pattern in the lyophobic region is summarized. Figure 2a-d shows isolated large droplets on the lyophilic region surrounded by a depletion zone, which becomes smaller with increasing gas flow. In fig. 2e-h, we see the pattern that develops under the same conditions, when the lattice constant of the quadratically ordered lyophilic circles is smaller. Figure 2i-l shows a schematic drawing of the depletion zones around this lattice of lyophilic patches. The extent of the depletion zones has been taken from fig. 2a-d, respectively.

In fig. $2 \mathrm{e}$ the depletion zone is so large that the droplets only condense on the lyophilic region. The lyophobic parts remain free of droplets. In the schematic drawing (fig. 2i) it can be seen that the depletion zones strongly overlap, and just a small area in the middle remains free. This is obviously not enough to induce nucleation and growth. Figure $2 \mathrm{f}$ shows the droplet pattern at an increased gas flux. A few small droplets condense in the lyophobic areas between the large droplets. It can be seen in the schematic drawing (fig. $2 \mathrm{j}$ ) that the depletion zones along the lattice axes almost touch each other, but there is enough space along the diagonal for droplets to nucleate. In fig. $2 \mathrm{~g}$ few droplets nucleate along the axes but many in between the elementary cell. Their number decreases with time due to coalescences. The distance between neighboring depletion zones is larger (fig. 2k). In fig. $2 \mathrm{~h}$ the depletion zones are so small that the large droplets are surrounded completely by small droplets on the lyophobic area (fig. 2l). They grow via the same processes as on the unstructured area (growth of single droplets and coalescences). 


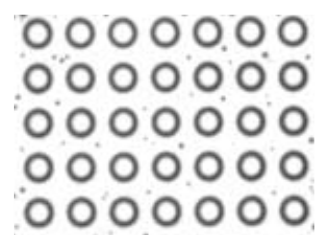

$2 \mathrm{~s}$

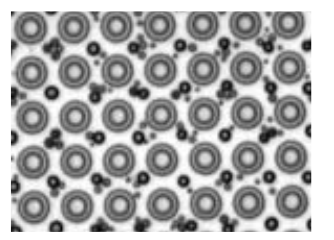

$8 \mathrm{~s}$

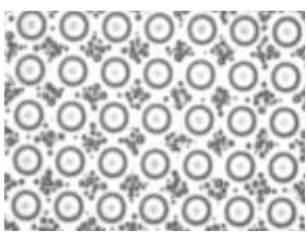

$4 \mathrm{~s}$

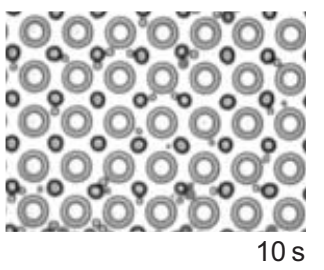

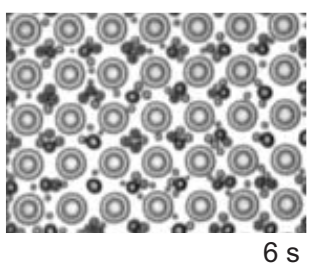

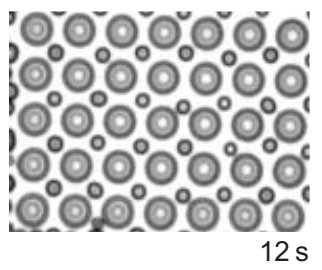

Fig. 5 - Substructure formation can be induced by overlapping depletion zones. Temporal development of a droplet pattern on chemically structured surfaces. Shown is a characteristic part of the substrate of droplets condensed on quadratically ordered lyophilic circles with diameter $d=5 \mu \mathrm{m}$ and lattice constant $a=6 \mu \mathrm{m}$ (upper part $a=9 \mu \mathrm{m}$ ). Different samples have been exposed to the vapor for the indicated amount of time and investigated. For each time and each repetition a freshly prepared samples was used. In the $t=12 \mathrm{~s}$ image a regularly ordered substructure has developed in between each droplet.

When the depletion zones are only slightly smaller than the lattice constant, a formation of substructures can be observed. In fig. 5 the temporal development of the droplet pattern is presented. It shows typical images of a series of different samples (lyophilic circles with $5 \mu \mathrm{m}$ diameter and $6 \mu \mathrm{m}$ lattice constant), which were exposed to vapor for different amounts of time ( 2 to $12 \mathrm{~s}$ ). After $2 \mathrm{~s}$, the lyophilic circles are completely wet and on the lyophobic region some droplets have nucleated and grown (or are already evaporated again before taking the picture). After $4 \mathrm{~s}$, a lot of droplets nucleated in the middle of the elementary cell of the lattice and some of them coalesced. Along the lattice axes almost no droplets exist. The droplets in the middle grow further after $6 \mathrm{~s}$ and $8 \mathrm{~s}$, and, on the one hand their mean radius increases, while on the other hand, their number decreases due to coalescences. After $10 \mathrm{~s}$, only few droplets on the lyophobic part remain while after $12 \mathrm{~s}$ it is just one droplet, which develops on the interstitial lattice sites. A substructure develops consisting of unpinned droplets on well-defined areas in the unstructured region. If the condensation proceeds, this substructure remains stable until the droplets reach a size such that they touch the pinned droplet and coalesce.

This effect of substructure formation is quite robust against variation of parameters. But it is necessary that the depletion zones are small enough in order to nucleate at least some droplets on the interstitial sites. If the depletion zones are small compared to the lattice constant, no substructure formation is observed. For larger depletion zones we observed this substructure formation also for diameter $d=5 \mu \mathrm{m}$ and lattice constant $a=9 \mu \mathrm{m}$, as well as for $d=$ $10 \mu \mathrm{m}$ and $a=15 \mu \mathrm{m}$ or $18 \mu \mathrm{m}$. The substructures also occur for different geometries. We also observed them on triangular patterns as well as on lyophobic circles in a lyophilic environment.

The described phenomenon of substructure formation is a consequence of fundamental growth mechanisms and could also occur in other systems, e.g. in the case of submonolayer growth, where capture zones are used to describe the growth of islands [14]. It should also be taken into account when breath figures are used to characterize artificially produced wetting structures [5].

In summary, it has been shown that during a breath figure experiment on chemically struc- 
tured substrates depletion zones develop around lyophilic patches, where no further nucleation and growth of droplets occurs. The size of the depletion zones depends on the incoming gas flow as well as on the contact angle of the lyophobic substrate. If the depletion zones of neighboring droplets overlap, different scenarios of condensation pattern formation in the lyophobic regions are observed. For larger depletion zones compared to the lattice constant of the underlying wetting structure, no droplets nucleate and grow in the lyophobic region. For intermediate depletion zones, one single droplet develops more or less on the interstitial sites leading to a regularly ordered substructure. For smaller depletion zones, less ordered structures are obtained. With this mechanism of structure formation droplets can be prepared on well-defined regions in unstructured areas by using a rougher prestructure and working in the intermediate regime of the range of the depletion zones.

$$
* * *
$$

We gratefully acknowledge financial support from the Deutsche Forschungsgemeinschaft, SFB 513.

\section{REFERENCES}

11] Beysens D., Steyer A., Guenoun P., Fritter D. and Knobler C. M., Phase Transit., 31 (1991) 219.

[2] Beysens D. and Knobler C. M., Phys. Rev. Lett., 57 (1986) 1433.

[3] Fritter D., Knobler C. M. and Beysens D. A., Phys. Rev. A, 43 (1991) 2858.

[4] ZhaO H. and Beysens D., Langmuir, 11 (1995) 627.

[5] Lopez G. P., Biebuyck H. A., Frisbie C. D. and Whitesides G. M., Science, 260 (1993) 647.

[6] Kumar A. and Whitesides G. M., Science, 293 (1994) 60.

[7] Aizenberg J., Black A. J. and Whitesides G. M., J. Am. Chem. Soc., 121 (1999) 4500.

[8] Lide D. R. (Editor), Handbook of Chemistry and Physics, 79th edition (CRC Press LLC, Boca Raton, Florida) 1998.

[9] Kumar A. and Whitesides G. M., Appl. Phys. Lett., 63 (1993) 2002.

[10] Kumar A., Biebuyck H. A. and Whitesides G. M., Langmuir, 10 (1994) 1498.

[11] Kim E. and Whitesides G. M., J. Phys. Chem. B, 101 (1997) 855.

[12] Steyer A., Guenoun P., Beysens D. and Knobler C. M., Phys. Rev. A, 44 (1991) 8271.

[13] Sigsbee R. A., Nucleation, edited by Zettlemoyer A. C. (Marcel Dekker, Bethlem, PA) 1969, p. 151.

[14] Mulheran P. A. and Blackman J. A., Phys. Rev. B, 53 (1996) 10261. 